\title{
Passive Bilateral Control of a Teleoperation System Considering Hysteresis Nonlinearity of Slave Robot
}

\author{
Reza Seifabadi ${ }^{1}$, Seyed Mehdi Rezaei ${ }^{1}$, Saeed Shiry ${ }^{2}$, Mozafar Saadat ${ }^{3}$, \\ Mohammad Zarei-nejad ${ }^{1}$, Kamran Razi ${ }^{4}$, and Hossein Habibollahi ${ }^{1}$ \\ ${ }^{1}$ Amirkabir University of Tech., Mechanical Engineering Dept. \& \\ New Technology Research Centre (NTRC) \\ ${ }^{2}$ Amirkabir University of Tech., Computer Engineering Dept., \\ ${ }^{3}$ University of Birmingham, Mechanical and Manufacturing Engineering Dept., \\ ${ }^{4}$ University of Tehran, Electrical and Computer Engineering Dept., Tehran, Iran \\ \{rezaseifabadi, smrezaei, mzare, shiry\}@aut.ac.ir, \\ hhabibollahi@gmail.com, k.razi@ece.ut.ac.ir, m.saadat@bham.ac.uk
}

\begin{abstract}
The slave robot of the macro-micro teleoperation system presented in this paper is a 1-DOF piezo actuator including hysteresis nonlinearity. This nonlinear behavior makes robot control a complex task. In this research the nonlinear and uncertain dynamics of the slave robot has been entered directly into the teleoperation control loop. The LuGre friction model is used as the estimator of the hysteresis loop to cancel out this undesirable term. A 2-DOF master-slave system is decomposed into two 1-DOF systems: a shape system representing the master-slave position coordination, and a locked system representing the dynamics of the coordinated system. For making the closed-loop teleoperation system passive against dynamic parameter uncertainty and force measurement inaccuracy, four virtual flywheels are designed. In this way, the energy generated by troublesome terms inside controllers (i.e. the terms which may endanger passivity of the controller) would be taken from the bounded kinetic energy deposited on these flywheels. Simulations are performed to show effectiveness of the proposed controllers.
\end{abstract}

Keywords: macro-micro telemanipulation, LuGre model, decomposition, shape and locked system, virtual flywheel, robust passivity, scaling, Hysteresis.

\section{Introduction}

In teleoperation, there are many cases in which slave environment is in micrometer dimensions. The operation complexity urges human operator to be present in the control loop. Operator interacts with a macro-scaled master robot, say a joystick, whereas slave robot interacts with a micro-scaled environment. Micro-assembly [1] and In Vitro Fertilization [2] are two exemplary applications of such systems. In micromanipulation, the Piezo-actuator based slave robots have been noticed due to their ability in high precision positioning [3]. 
The most important drawback in application of piezo-actuators is their nonlinear hysteresis behavior, making their control complex [4], [5]. In this research, the nonlinear dynamics of the slave robot has been entered directly into the teleoperation control loop. The LuGre friction model is used as the estimator of the hysteresis loop to cancel out this undesirable term. This model is used for some reasons: 1) it is proved that the model estimates the hysteresis loop of the piezo-actuator precisely [6]. 2) Hysteresis effect of piezo-positioning mechanism can be separately added to slave linear dynamics [6]. Then it is possible to linearize the system easily. 3) The conditions under which LuGre friction model satisfies passivity condition are available [7]. This ability says if the hysteresis compensating term of the controller is passive.

A 2-DOF master-slave system is decomposed to two 1-DOF systems: a shape system representing the master-slave position coordination, and a locked system representing the dynamics of the coordinated system. This approach was proposed firstly by Li for Linear Dynamically Similar (LDS) teleoperation system [8] and then developed to a general $2 n$-DOF nonlinear system [9] whose nonlinearity was caused by the coupling of degrees of freedom. Because of this non-intrinsic nonlinearity, $\dot{M}_{i}-2 C_{i}$ (defined later) were skew-symmetric. This property brings a great deal of convenience during both control design and stabilization discussion. Instead, the nonlinearity here belongs to the hysteretic nature of the piezo-actuator. Because of this substantial change, almost all of theorems, propositions, control laws, etc. inside [10], [9] remains debatable when they are applied to our system. In fact, the main contribution of this article is to reestablish this powerful approach for the hysteresis-type nonlinear teleoperated systems. Also, robust passivity of the macro-micro teleoperator is guaranteed in the presence of scaling factors and LuGre model parameter uncertainties.

A shape system controller is designed to achieve position coordination in the presence of arbitrary human/environment force. After the master-slave position coordination occurred, the locked system controller induces a desired dynamics to the locked system. For making the closed-loop teleoperator passive against dynamic parameter uncertainty and force measurement inaccuracy, a negative semi-definite (NSD) structure is implemented for the designed controller. Inside the controllers, there are troublesome terms (such as feedforward cancelation term in shape system control) that endanger the passivity of the system. For solving this problem, virtual flywheel concept has been used. This approach, a corresponding approach to the passivity observer approach [11], was introduced by $\mathrm{Li}$ in [12] and then by Lee in [10], [9]. In this way, the energy generated by troublesome terms inside controllers would be taken from the bounded kinetic energy deposited on the flywheels. Unlike previous works which used two flywheels, four virtual flywheels are used here to minimize performance degradation when either of flywheels depletes energy.

In section 2 of this paper, the master and slave robots of the current teleoperator are modeled. In section 3 , the problem is defined and the control objectives are introduced. In section 4, the system is decomposed to the locked and shape system. Section 5 designs the locked and shape controller to satisfy objectives. In section 6 , passivity of the designed controllers is discussed. In section 7, dynamic parameters are set to establish simulation. Section 8 verifies veracity of the control design. Section 9 contains some concluding remarks. 


\section{Teleoperator Modeling}

\subsection{Dynamic Modelling for the Master Robot}

The master robot is a single degree of freedom mass-damper system.

$$
M_{1} \ddot{q}_{1}+C_{1} \dot{q}_{1}=T_{1}+F_{1}
$$

Where $q_{l}$ denotes master position, $M_{l}$ and $C_{l}$ denote the inertia and viscous damping coefficient of the master, $F_{l}$ denotes the force applied at the master side by the operator and $T_{l}$ is the master control signal.

\subsection{Dynamic Modelling for the Slave Robot}

The slave robot is a 1-DOF piezo-stage with hysteresis behavior. The hysteresis friction model, called LuGre model, is used due to the mentioned reasons and those which are explained in [6].

The linear slave robot is modified to describe hysteresis effect of the piezo-actuator by separately adding a load term $F_{H}\left(\dot{q}_{2}\right)$.

$$
M_{2} \ddot{q}_{2}+C_{2} \dot{q}_{2}+F_{H}\left(\dot{q}_{2}\right)=T_{2}+F_{2}
$$

$q_{2}$ is the salve position, $M_{2}$ and $C_{2}$ are inertia and viscous coefficient, $T_{2}$ denotes the salve control law. $F_{2}$ is the force exerted by the environment to the slave, and $F_{H}\left(\dot{q}_{2}\right)$ is calculated from (3):

$$
F_{H}\left(\dot{q}_{2}\right)=\sigma_{0} \bar{z}-\frac{\sigma_{1}}{h\left(\dot{q}_{2}\right)} \bar{z}\left|\dot{q}_{2}\right|+\left(\sigma_{1}+\sigma_{2}\right) \dot{q}_{2}
$$

where

$$
\sigma_{0} h\left(\dot{q}_{2}\right)=f_{C}+\left(f_{S}-f_{C}\right) \cdot e^{-\left(\dot{q}_{2} / \dot{x}_{S}\right)^{2}}
$$

Here $\sigma_{0}, \sigma_{1}, \sigma_{2}, f_{C}, f_{S}$, and, $\dot{x}_{S t}$ are constants. $\bar{z}$ is gained from (5):

$$
\frac{d \bar{z}}{d t}=\dot{q}_{2}-\frac{\left|\dot{q}_{2}\right|}{h\left(\dot{q}_{2}\right)} \bar{z}
$$

For more details about LuGre friction model, refer to [13] and [6].

\subsection{Position and Power Scaling}

In the macro-micro teleoperation systems, power and position levels in both sides are substantially different. Therefore, scaling of these signals should be concerned when they are transmitted through the communication channels. Both power and position scaling are applied to the master side. Here is the modified master dynamics:

$$
\rho\left(\frac{M_{1}}{\alpha} \ddot{Q}_{1}+\frac{C_{1}}{\alpha} \dot{Q}_{1}=T_{1}+F_{1}\right)
$$

$\rho$ and $\alpha$ are user-specified power and position scaling factors, respectively. $Q_{l}$ is the scaled master position $q_{1}$ (i.e. $Q_{l}=\alpha q_{1}$ ). 


\section{Problem Formulation}

\subsection{Generality}

LuGre friction model is said to satisfy the energetic passivity condition if there exists a finite constant $b \in R$ such that (s.t) for $t \geq 0$ :

$$
\int_{0}^{t} s_{L}\left(\dot{q}_{2}(\tau)\right) d \tau=\int_{0}^{t}\left[F_{H}\left(\dot{q}_{2}(\tau)\right) \dot{q}_{2}(\tau)\right] d \tau \leq b^{2}
$$

where $s_{L}\left(\dot{q}_{2}\right)$ is the energy generated by the LuGre model estimator $F_{H}\left(\dot{q}_{2}\right)$ inside the teleoperator. Equation (7) is true if the following inequality occurs [7]:

$$
\frac{1}{f_{C}} \leq \frac{1}{f_{S}}\left(1+\frac{\sigma_{2}}{\sigma_{1}}\right)
$$

To deliver a linear system, it is desirable to cancel the nonlinear term $F_{H}\left(\dot{q}_{2}\right)$ out of the system. However, exact cancellation is not feasible since an estimation of this term is only available. This poses some inconveniences while proving of the proposition 1 .

Slave controller $T_{2}$ can be rewritten as following:

$$
T_{2}=T_{2}^{\prime}+\hat{F}_{H}\left(\dot{q}_{2}\right)+\tilde{F}_{\max } \cdot \operatorname{sgn}\left(\dot{q}_{2}\right)
$$

Here, $T_{2}^{\prime}$ is a portion of the slave controller $T_{2}$ to achieve other objectives which will be introduced in future. $\hat{F}_{H}\left(\dot{q}_{2}\right)$ is the best estimation of the nonlinear term. The last term is introduced to guarantee the passivity of the system after the nonlinear term is cancelled out. If (8) is substituted to (2), the result is:

$$
M_{2} \ddot{q}_{2}+C_{2} \dot{q}_{2}+\widetilde{F}_{H}\left(\dot{q}_{2}\right)+\tilde{F}_{\text {max }} \cdot \operatorname{sgn}\left(\dot{q}_{2}\right)=T_{2}^{\prime}+F_{2}
$$

where $\tilde{F}_{H}\left(\dot{q}_{2}\right)=F_{H}\left(\dot{q}_{2}\right)-\hat{F}_{H}\left(\dot{q}_{2}\right)$ and $\tilde{F}_{\max } \geq\left|F_{H}\left(\dot{q}_{2}\right)-\hat{F}_{H}\left(\dot{q}_{2}\right)\right|, \forall t \geq 0$.

If the inequality (7-1) is checked for the parameters listed in Table 1, it is seen that the LuGre model of the slave dynamics i.e. $F_{H}\left(\dot{q}_{2}\right)$ is an active operator (does not satisfy (7)). Thus, compensating term of the controller i.e. $\hat{F}_{H}\left(\dot{q}_{2}\right)$ in (8) will be a passive operator. Therefore, it is enough to ensure passivity of $T^{\prime}{ }_{2}$ while discussing the passivity of the proposed controller in section 6 .

Definitions of a passive teleopeator and a passive controller could be provided similar to (9) and [9].

Proposition 1: Controller passivity implies energetic passivity of the teleoperator.

Proof is omitted for brevity.

Proposition 1 enables us concentrate just on the controller passivity without concerning about the teleoperator passivity.

\subsection{Control Objectives}

A controller should be designed to satisfy the following objectives: 
1) The closed-loop teleoperator robustly satisfy the energetic passivity condition.

2) Positions of the master and the slave robots are coordinated perfectly. That means $\forall\left(F_{1}, F_{2}\right)$,

$$
q_{E}=Q_{1}-q_{2} \rightarrow 0
$$

$q_{E}$ is the position error between two robots. Once the coordination is achieved, the teleoperator system would have the following dynamics:

$$
M_{L} \ddot{q}_{L}(t)+C_{L} \dot{q}_{L}(t)+K_{L}(t)=T_{L}+F_{L}
$$

where

$$
\begin{aligned}
& M_{L}=\rho \frac{M_{1}}{\alpha}+M_{2}, \quad C_{L}=\rho \frac{C_{1}}{\alpha}+C_{2}, F_{L}=\rho F_{1}+F_{2}, T_{L}=\rho T_{1}+T_{2}, \\
& K_{L}(t)=\tilde{F}_{H}\left(\dot{q}_{L}\right)+\tilde{F}_{\text {max }} \cdot \operatorname{sgn}\left(\dot{q}_{L}\right)
\end{aligned}
$$

and $\dot{q}_{L}(t)=\dot{Q}_{1}(t)=\dot{q}_{2}(t)$ when $q_{E}=Q_{1}-q_{2} \rightarrow 0$.

3) The coordinated teleoperator dynamics (11) converges to the following desired dynamics:

$$
M_{L}^{\prime} \ddot{q}_{L}(t)+C_{L}^{\prime} \dot{q}_{L}(t)+K_{L}^{\prime}=F_{L}
$$

$M_{L}^{\prime}, C_{L}^{\prime}$, and $K_{L}^{\prime}$ are designed to induce high dexterity to the operator.

\section{The Non-passive Decomposition}

The transformer $S$ is proposed for the 2-DOF teleoperator dynamics which decomposes the system into two (not necessarily) decoupled systems: The shape system, representing the master-slave coordination aspect; and the locked system, describing overall motion of the coordinated teleoperator. The decomposition is designed based on the facts mentioned in [9]:

Then, the following transformation is proposed to achieve the mentioned goals:

$$
\left[\begin{array}{l}
V_{L} \\
q_{E}
\end{array}\right]=\underbrace{\left[\begin{array}{cc}
x & y \\
1 & -1
\end{array}\right]}_{=: S}\left[\begin{array}{l}
Q_{1} \\
q_{2}
\end{array}\right]
$$

Condition 3) is directly achieved from definition (14). $x$ and $y$ satisfying the other two conditions are as following:

$$
x=\frac{\rho M_{1}}{\rho M_{1}+M_{2} \alpha}, \quad y=\frac{M_{2} \alpha}{\rho M_{1}+M_{2} \alpha}
$$

Using the transformer $S$, the following partially decomposed dynamics will be achieved:

$$
\begin{aligned}
& \underbrace{M_{L} \ddot{q}_{L}(t)+C_{L} \dot{q}_{L}(t)}_{\text {Locked system dynamics }}+\underbrace{C_{L E} \dot{q}_{E}(t)}_{\text {coupling }}+K_{L}(t)=T_{L}+F_{L} \\
& \underbrace{M_{E} \ddot{q}_{E}(t)+C_{E} \dot{q}_{E}(t)}_{\text {Shaped system dynamics }}+\underbrace{C_{E L} \dot{q}_{L}(t)}_{\text {coupling }}+K_{E}(t)=T_{E}+F_{E}
\end{aligned}
$$


where

$$
\begin{gathered}
C_{L E}=\frac{\rho C_{1} M_{2}-\rho M_{1} C_{2}}{\rho M_{1}+M_{2} \alpha}, C_{E L}=C_{L E}, C_{E}=\frac{\rho C_{1} C_{2}}{\rho C_{1}+C_{2} \alpha}, M_{E}=\frac{\rho M_{1} M_{2}}{\rho M_{1}+M_{2} \alpha}, \\
K_{E}=-\frac{\rho M_{1}}{\rho M_{1}+M_{2} \alpha} K_{L}, T_{E}=\frac{\rho\left[T_{1} M_{2} \alpha-M_{1} T_{2}^{\prime}\right]}{\rho M_{1}+M_{2} \alpha}, F_{E}=\frac{\rho\left[F_{1} M_{2} \alpha-M_{1} F_{2}\right]}{\rho M_{1}+M_{2} \alpha} .
\end{gathered}
$$

In comparison with the decomposed system presented in [9], two following decisive properties are not satisfied for the current decomposed system:

1) $\dot{M}_{L}-2 C_{L}$ and $\dot{M}_{E}-2 C_{E}$ are skew symmetric 2$) C_{L E}+C_{E L}=0$

Since these two properties are not satisfied, the approach discussed in [9] is not applicable for the current teleoperator. One disappointing result is that the decomposition now is not passive. The proof is denied here for brevity.

\section{Control Design}

This section aims to achieve the control objectives introduced in section 3.2. For this reason, $T_{L}$ and $T_{E}$ in (16) and (17) should be designed properly.

\subsection{Coordination Control}

The objective here is that $q_{E}=Q_{1}-q_{2} \rightarrow 0$ in the presence of arbitrary human/environment force (i.e. $\forall\left(F_{1}, F_{2}\right)$ ). For this, feedforward cancellation should be taken into consideration. The following PD controller is proposed to satisfy the objective:

$$
T_{E}=-K_{v} \dot{q}_{E}-K_{p} q_{E}-\hat{F}_{E}(t)+\hat{C}_{E L} \dot{q}_{L}+\hat{C}_{E} \dot{q}_{E}
$$

Proposition 2: Suppose that $F_{1}, F_{2}, \dot{Q}_{1}$, and $\dot{q}_{2}$ are bounded. If the master and slave dynamics parameters and force measurements $\left(F_{l}\right.$ and $\left.F_{2}\right)$ are accurate, then the shape system will asymptotically converge to the equilibrium point $\left(q_{E}, \dot{q}_{E}\right)=(0,0)$. If feedforward cancelation is not used, or its estimation error $\widetilde{F}_{E}=F_{E}-\hat{F}_{E}$ is bounded, or dynamic parameters of the robots involve uncertainty, then $\left(q_{E}, \dot{q}_{E}\right)$ is ultimately bounded. Proof is omitted for brevity.

\subsection{Locked System Control}

The objective here is to replace locked system dynamics (16) with the desired dynamics (13) under the locked system control $T_{L}$. The control law that satisfies this objective is:

$$
T_{L}=\left(\frac{M_{L}}{M_{L}^{\prime}}-1\right) F_{L}+\left(C_{L}-C_{L}^{\prime} \frac{M_{L}}{M_{L}^{\prime}}\right) \dot{q}_{L}+\left(K_{L}-K_{L}^{\prime} \frac{M_{L}}{M_{L}^{\prime}} q_{L}\right)+C_{L E} \dot{q}_{E}
$$




\section{Passive Control Implementation}

\subsection{Passive Control Implementation}

Two shaped and locked system control architecture can be rewritten in a matrix form:

$$
\left[\begin{array}{c}
T_{L} \\
T_{E}
\end{array}\right]=\left[\begin{array}{cc}
C_{L}-C_{L}^{\prime} \frac{M_{L}}{M_{L}^{\prime}} & C_{L E} \\
C_{L E} & C_{E}-K_{v}
\end{array}\right]\left[\begin{array}{l}
\dot{q}_{L} \\
\dot{q}_{E}
\end{array}\right]+\left[\begin{array}{c}
-K_{L}^{\prime} \frac{M_{L}}{M_{L}^{\prime}} q_{L} \\
-K_{p} q_{E}
\end{array}\right]+\left[\begin{array}{c}
\left(\frac{M_{L}}{M_{L}^{\prime}}-1\right) F_{L}+K_{L} \\
-\hat{F}_{E}
\end{array}\right]
$$

It is possible to detect (potentially) active terms inside controllers by multiplying both sides by $\left[V_{L} \dot{q}_{E}\right]$ (omitted for brevity). By this method, we come to the conclusion that $C_{\mathrm{L}}, C_{\mathrm{E}}, C_{\mathrm{LE}}, C_{\mathrm{EL}}$ and the terms in the last matrix of (22) are (potentially) active. Now, to enforce passivity of the control architecture, terms that may endanger passivity needs to be bounded. For this reason, fictitious energy storage elements (with flywheel dynamics) are used in the controller s.t the energy generated by terms I and IV of (26) are taken from these flywheels. In this way, the energy generated by part I and IV of (26) would be bounded by the energy deposited on the flywheels.

The following four 1-DOF fictitious flywheels are presented: ${ }^{{ }} M_{f}{ }_{f}{ }^{L_{f}}{ }_{f}={ }^{L_{f}} T_{f}$ (locked system flywheels), and ${ }^{E_{i}} M_{f}{ }_{i} \ddot{x}_{f}={ }^{E_{i}} T_{f}$ (shape system flywheels). The total control (22) is established using the following Negative Semi Definite (NSD) implementation structure:

$$
\left[\begin{array}{c}
T_{L} \\
T_{E} \\
{ }^{L_{1}} T_{f} \\
L_{2} T_{f} \\
E_{1} T_{f} \\
E_{2} T_{f}
\end{array}\right]=\underbrace{\left[\begin{array}{cccccc}
\Gamma_{d}(t) & 0 & \Pi_{\varphi}^{1}(t) & \Pi_{\varphi}^{2}(t) & 0 & 0 \\
0 & \Delta_{d}(t) & 0 & 0 & \Sigma_{E}^{1}(t) & \Sigma_{E}^{2}(t) \\
-\Pi_{\varphi}^{1}(t) & 0 & 0 & 0 & 0 & 0 \\
-\Pi_{\varphi}^{2}(t) & 0 & 0 & 0 & 0 & 0 \\
0 & -\Sigma_{E}^{1}(t) & 0 & 0 & 0 & 0 \\
0 & -\Sigma_{E}^{2}(t) & 0 & 0 & 0 & 0
\end{array}\right]}_{\Omega(t)}\left[\begin{array}{c}
V_{L} \\
\dot{q}_{E} \\
L_{1} \dot{x}_{f} \\
L_{2} \\
\dot{x}_{f} \\
E_{1} \dot{x}_{f} \\
E_{2} \dot{x}_{f}
\end{array}\right]+\left[\begin{array}{c}
-K_{L}^{\prime} \frac{M_{L}}{M_{L}^{\prime}} q_{L} \\
-K_{p} q_{E} \\
0 \\
0 \\
0 \\
0
\end{array}\right]
$$

The proof why this NSD implementation is intrinsically passive is omitted for brevity.

\subsection{Design of NSD Implementation Parameters}

The entries $\Pi_{\varphi}^{i}, \Sigma_{E}^{i}, \Gamma_{d}(t)$, and $\Delta_{d}(t)$ in (23) should be now designed such that the control architecture (22) is duplicated:

$$
\begin{aligned}
& \text { i) } \Gamma_{d}(t)=-\left[1-g\left({ }^{{ }^{4}} \dot{x}_{f}\right)^{L_{1}} \dot{x}_{f}\right] C_{L}^{\prime} \frac{M_{L}}{M_{L}^{\prime}}, \text { ii) } \Pi_{\varphi}^{1}(t)=\left(\frac{M_{L}}{M_{L}^{\prime}}-1\right) F_{L} \cdot g\left({ }^{L_{1}} \dot{x}_{f}\right)_{L}-\underbrace{C_{L}^{\prime} \frac{M_{L}}{M_{L}^{\prime}} V_{L} \cdot g\left({ }^{L_{1}} \dot{x}_{f}\right)}_{\text {energy recapture }} \\
& \text { iii) } \left.\Pi_{\varphi}^{2}(t)=\left(K_{L}+C_{L} V_{L}+C_{L E} \dot{q}_{E}\right) \cdot g\left({ }^{L_{2}} \dot{x}_{f}\right), \text { iv }\right) \Delta_{d}(t)=-\left[1-g\left({ }^{E_{1}} \dot{x}_{f}\right)^{E_{1}} \dot{x}_{f}\right] K_{v} \\
& \text { v) } \Sigma_{E}^{1}(t)=\underbrace{-g\left({ }^{E} \dot{x}_{f}\right) K_{v} \dot{q}_{E}}_{\text {energy recapture }}+\frac{P_{1}(t)}{E_{1} \dot{x}_{f}}\left(-\hat{F}_{E}\right) \text {, vi) } \Sigma_{E}^{2}(t)=\frac{P_{2}(t)}{E_{2} \dot{x}_{f}}\left(C_{E L} \dot{q}_{L}+C_{E} \dot{q}_{E}\right)
\end{aligned}
$$


The initial velocity of flywheels under which the locked and shaped systems do not deplete energy for $t>0$, are discussed in [10].

Theorem 1: The decomposed mechanical teleoperator (16) and (17) under the NSD implementation (23) and (24) is considered.

1) The closed loop teleoperator is energetically passive (i.e. satisfies (10)), even in the presence of inaccurate force sensing $F_{1}$ and $F_{2}$ and dynamic parameter uncertainty.

2) Consider the teleoperation system free from inaccuracy of force sensing $F_{l}$ and $F_{2}$ and dynamic parameter uncertainty. If $\left|E_{1} \dot{x}_{f}\right| \geq f_{o}$ and flywheels are started with appropriate initial velocities, then $\left(q_{E}, \dot{q}_{E}\right) \rightarrow(0,0)$ exponentially. That is, $\dot{q}_{L}(t)=\dot{Q}_{1}(t)=\dot{q}_{2}(t)$.

3) Consider the teleoperation system under all the assumptions presented in 2). If $\left|{ }^{L_{i}} \dot{x}_{f}\right| \geq f_{o}$ for $\forall t \geq 0$, then the target dynamics (13) is achieved.

4) Suppose the situation in which either (maybe all) of the virtual flywheels is switched off. The closed- loop teleoperator will still remain energetically passive. Proof is omitted for brevity.

\section{Dynamic Parameter Design}

Dynamic parameters of the master and slave robots, i.e. $M_{1}, C_{1}$ and $M_{2}, C_{2}$ and scaling factors $\alpha$ and $\rho$, are chosen with respect to our specific micromanipulation task. The master robot is supposed to be a 1-DOF DC motor. The slave robot, as introduced before, is a 1 -DOF linear piezo-actuator with a maximum $100 \mu \mathrm{m}$ movement. The position scaling factor $\alpha$ is designed so that 90 degrees of master rotation causes

Table 1. Designed Parameters

\begin{tabular}{cccccccc}
\hline Symbol & $\begin{array}{c}\text { Quantity } \\
(S I)\end{array}$ & Symbol & $\begin{array}{c}\text { Quantity } \\
(S I)\end{array}$ & Symbol & $\begin{array}{c}\text { Quantity } \\
(S I)\end{array}$ & Symbol & $\begin{array}{c}\text { Quantity } \\
(S I)\end{array}$ \\
\hline$\alpha$ & $111.1^{-1}$ & $\varepsilon$ & 0.1 & $\hat{\sigma}_{2}$ & 1 & $C_{L}^{\prime}$ & 25 \\
$\rho$ & $10^{-1}$ & $\tilde{F}_{\max }$ & 5 & $\hat{f}_{C}$ & 0.8 & $K_{L}^{\prime}$ & 0 \\
$C_{1}$ & 4 & ${ }^{L_{i}} M_{f}$ & 1 & $\hat{f}_{S}$ & 2 & $K_{v}$ & 0.5 \\
$C_{2}$ & 0.015 & ${ }^{E_{i}} M_{f}$ & 1 & $\hat{\dot{x}}_{S t}$ & 0.003 & $K_{p}$ & 500 \\
$M_{1}$ & 1 & ${ }^{E_{1}} f_{o}$ & $1(3)$ & $\hat{F}_{E \max }$ & 100 & $f_{C}$ & 1 \\
$M_{2}$ & 1 & ${ }^{4} \dot{x}_{f}(0)$ & 3 & $A_{\max }$ & 1 & $f_{S}$ & 1.5 \\
$\hat{C}_{1}$ & 2.8 & $L_{{ }_{2}} \dot{x}_{f}(0)$ & 4 & $\delta_{v}$ & 5000 & $\dot{x}_{S t}$ & 0.001 \\
$\hat{C}_{2}$ & 0.035 & $\sigma_{0}$ & $10^{\wedge} 5$ & $\bar{G}_{L}$ & 2 & $\hat{\sigma}_{0}$ & $1.2 \times 10^{5}$ \\
$\hat{M}_{1}$ & 0.85 & $\sigma_{1}$ & $\sqrt{10 \wedge}$ & $V(0)$ & $5 \times 10^{-4}$ & $\hat{\sigma}_{1}$ & $1.25 \times \sqrt{10^{5}}$ \\
$\hat{M}_{2}$ & 1.1 & $\sigma_{2}$ & 0.4 & ${ }^{4} f_{o}$ & 3 & ${ }^{L_{2}} f_{o}$ & 4 \\
$M_{L}^{\prime}$ & 2.2 & ${ }^{E_{2}} f_{o}$ & $1(3)$ & ${ }^{E_{1}} \dot{x}_{f}(0)$ & 4 & ${ }^{E_{2}} \dot{x}_{f}(0)$ & 2 \\
\hline
\end{tabular}


$100 \mu \mathrm{m}$ movement of the slave robot, when the human force is exerting at the length of $0.2 \mathrm{~m}$ from the DC motor pivot (almost $1 \mu \mathrm{m}$ of the slave motion per 1 degree of the master rotation). The power scaling $\rho$ is selected to be $1 / 10$. Therefore, the human power will be attenuated 10 folds when it arrives to the slave robot (force scaling of $\rho / \alpha)$. The constant characteristic parameters for describing the hysteresis loop of the piezo-positioning mechanism of the slave robot, i.e. $\sigma_{0}, \sigma_{1}, \sigma_{2}, f_{C}, f_{S}$, and $\dot{x}_{S t}$, are extracted from [6], which uses similar piezo-stage. Other parameters are designed according to discussed equations.

\section{Simulation Results}

In this section, the simulation results for the macro-micro teleoperation system are presented. To demonstrate the performance of the controller, the following scenario is organized; the human operator is modeled by a PD position tracking controller using spring and damping gains $70 \mathrm{~N} / \mathrm{m}$ and $50 \mathrm{~N} . \mathrm{s}$, respectively. At the $0-5$ second interval, the master robot is stabilized at the position $1 \mathrm{~cm}$. Then, at $5-12 \mathrm{~s}$, the master robot is pushed to a new position. While moving the robot to this target, the operator realizes existence of a deflectable object. The object is modeled by a $200 \mathrm{~N} / \mathrm{m}$ linear spring. Finally, at $12-20 \mathrm{~s}$, the human operator retracts the master to $-0.5 \mathrm{~cm}$.
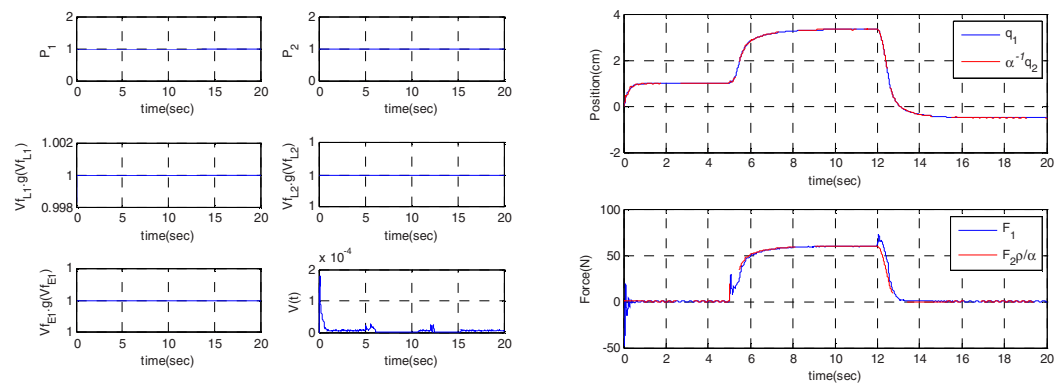

Fig. 1.

Fig. 2.

In Fig. 1 and Fig. 2, the teleoperator is involved with uncertainty, as introduced in Table 1. Human/environment forces are supposed to be free from inaccuracy. Therefore, dynamic parameter uncertainty is the only source of instability. All parameters are selected from Table 1. The following observations are achieved from these two figures: 1) As it is observed in Fig. 1, all flywheels are turn on. In other words, neither of flywheels depletes energy. Therefore, from part (2) of theorem 1, it is expected that $\left(q_{E}, \dot{q}_{E}\right) \rightarrow(0,0)$. Fig. 2 confirms this anticipation. 2) The teleoperation system is stable, even in the presence of dynamic parameter uncertainty. This validates robust passivity of the proposed controller, which is stated as part (1) of theorem 1. 3) $V(t)$ goes to zero, because of satisfactory position coordination of the master and slave robots (see Fig. 2). This validates correctness of proposition 2. 4) Desired objective 1, i.e. position tracking $\forall\left(F_{1}, F_{2}\right)$, is satisfied, according to Fig. 2. 5) Fig. 2 says that 
when the slave robot is pushing against the obstacle (5-12 s), the contact force is faithfully reflected to the human. 6) During free motion (0-5 and 12-20 sec), both human and environment forces are expected to be zero. Fig. 2 satisfies this expectation. However, $F_{1}$ always oscillates when the master velocity changes suddenly (at $\mathrm{t}=0,5$, and $12 \mathrm{sec}$ corresponding to the damping value of the operator model).

Uncertainty can really endanger passivity of the system. To see that, let the threshold values of the shape flywheel speeds ${ }^{E_{i}} f_{o}$ are increased from 1 to 3 . Due to dynamic uncertainty, shape system controller is going to be active. It consumes shape flywheels kinetic energy to produce this energy. Therefore, shape flywheels will drop sooner their speed below the threshold (in comparison with the initial values). Consequently, shape flywheels will deplete energy i.e., $P_{1}$ and $P_{2}$ become zero, based on the appropriate given initial velocity of the flywheels.Also, $E_{1} \dot{x}_{f} g\left({ }^{E_{1}} \dot{x}_{f}\right)=V f_{E 1} \cdot g\left(V f_{E 1}\right)$ decrease below unity. In other words, shape system controller $\Sigma_{E}^{i}$ will be switched off. Consequently, position coordination will degrade according to proposition 2. All of these anticipations are confirmed through Fig. 3 and Fig. 4.
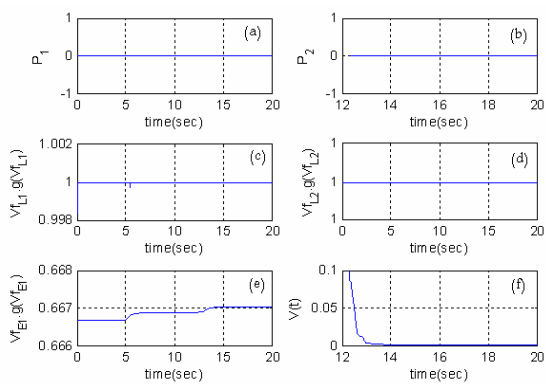

Fig. 3.
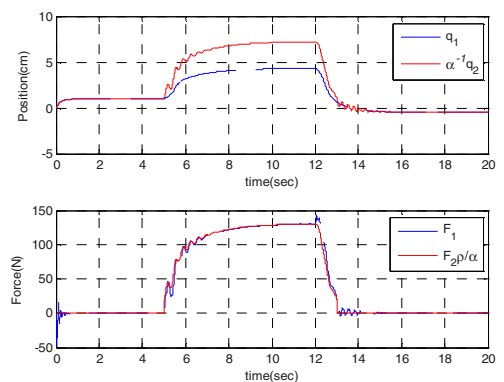

Fig. 4.

Although both shape flywheels are switched off, the system still remains stable. It means performance is sacrificed to keep the system passive. This fact supports validity of part (4) of theorem 1. It is reminded that human/environment force inaccuracy acts similar to the model uncertainty; therefore, it is not discussed here.

\section{Conclusions}

Because of both acceptable position and force tracking, this controller set-up can be used when the slave robot is interacting with a soft environment. Time delay is not taken into consideration throughout this research. This is based on the fact that two robots are supposed to be closed enough to each other and sampling time rates of our set-up are high enough. Nevertheless, authors are deeply interested in generalizing this control design to a delayed system as a future work. Also, this article lacks experimental verification. It is also desired to verify this control design through experiments. Due to limitations on the number of pages, the proofs and other complementary explanations 
have been omitted. Thus, a more complete version of this work or simulation files can be requested upon the reader's interest.

\section{References}

1. Kawaji, A., Arai, F., Fukuda, T.: Calibration for contact type of Micro-manipulation. In: Proc. of IEEE/RSJ Intern. Conf. on Intelligent Robotic, Korea, pp. 715-720 (1999)

2. Yu, S., Nelson, B.J.: Microrobotic cell injection. IROS, Seoul, Korea, pp.620-625 (2001)

3. Bergander, A., Breguet, J.M., Perez, R., Clavel, R.: PZT based manipulators for cell biology. In: Int. symp. on micROmechatronics and human science, Nagoya, japan, pp. 193 $196(2001)$

4. Mittal, G.S., Menq, C.H.: Hysteresis compensation in electromagnetic actuators through preisach model inversion. IEEE/ASME Trans.Mechatron 5(4), 394-409 (2000)

5. Hwang, L., Jan, C.: A reinforcement discrete neuro-adaptive control for unknown piezoelectric actuator systems with dominant hysteresis. IEEE Trans. Neural Netw. 14(1), 6678 (2003)

6. Lin, J., Shieh, H.J., Huang, P.K.: Adaptive Wavelet Neural Network Control with Hysteresis Estimation for Piezo-Positioning Mechanism. IEEE Trans. On Neural Networks 17(2) (March 2006)

7. Barabanov, N., Ortega, R.: Necessary and Sufficient Conditions for Passivity of the LuGre Friction Model. IEEE Transactions on Automatic Control 45(4) (April 2000)

8. Li, P.Y.: Passive control of bilateral teleoperated manipulators. In: Proc. Amer. Control Conf., pp.3838-3842 (1998)

9. Lee, D., Li, P.Y.: Passive bilateral control and tool dynamics rendering for nonlinear mechanical teleoperators. IEEE Trans. on Robotics 21(5) (October 2005)

10. Lee, D., Li, P.Y.: Passive bilateral feedforward control of linear dynamically similar teleoperated manipulators. IEEE Trans. on Robotics 19(3) (June 2003)

11. Hannaford, B., Ryu, J.: Time-domain passivity control of haptic interface. IEEE transaction. Robotics. Autom. 18(1), 1-10 (2002)

12. Li, P.Y., Horowitz, R.: Passive velocity field control of mechanical manipulators. IEEE Trans. on Robotics 15(4) (August 1999)

13. de Wit, C., Olsson, H., Aström, K.J., Lischinsky, P.: A new model for control of systems with friction. IEEE Trans. Autom. Control 40(3), 419-425 (1995) 